\title{
Open Information Structure Approach to Learning Support and Management with Kit-build Concept Map
}

\author{
Yusuke Hayashi \\ Department of Information Engineering \\ Graduate School of Engineering, Hiroshima University, Higashihiroshima, Japan \\ hayashi@lel.hiroshima-u.ac.jp
}

\begin{abstract}
Concept maps are a useful tool that encourages learners to explain their knowledge and understanding. From an educational perspective, the concept maps created by learners are a promising product for verifying student understanding. However, the diagnosis of conceptual maps created by learners remains an important issue for realizing educational interaction through conceptual maps. From a technology-enhanced learning perspective, several studies are already working on the automatic diagnosis of learner concept maps and provide appropriate feedback. The concept map for kit construction is a "closed-end" approach that allows automatic diagnosis of learner-generated concept maps. The learner creates a learner map with the parts that make up the concept map created by the teacher. This mechanism allows learners to construct concept maps and automatically evaluate learner maps for differences between learner and teacher concept maps. This paper provides an overview and case study of the KB map framework.
\end{abstract}

Keywords: concept map, open information structure approach, kit-build method, automated diagnosis, assessment

\section{INTRODUCTION}

Concept maps are a useful tool to encourage learners to explain their knowledge and understanding [1]. From an educational perspective, learner-created concept maps are a promising product for verifying student understanding [2][3]. However, the diagnosis of concept maps created by learners remains a significant issue for realizing educational interaction through concept maps. Learners may fail to build a proper concept map, and it is usually difficult for learners to recognize imperfections.

From a technology-enhanced learning perspective, several studies are already working on the automatic diagnosis of learner concept maps and providing appropriate feedback [4][5][6][7]. The basic approach to achieving assessment is to compare learner and teacher concept maps. Some surveys deal with automatic assessments and are specially designed to address cases where learners misspelled concepts based on natural language processing techniques or used concepts related to synonyms or appropriate concepts. Pay attention.

Another approach that enables the automated diagnosis of learner-generated concept maps is the "closed-end" approach [8]. In this approach, learners are provided with nodes and links that are composed of teacher concept maps, and learners must combine them to create concept maps [9][10]. The learners' concept maps are made up of the same elements as the teacher's map so that teachers can detect these differences.

The learner model and its diagnosis are shallow because learners can create maps within the limits of the parts provided. Therefore, they only deal with the recall and understanding level of Bloom's classification [11]. Also, the learner model is less accurate than if the element is drawn by the learner. However, due to the benefits of automated diagnostics, this approach has been adopted in some studies.

Hirashima et al. propose a concept map framework called "kit-build concept map" or "KB map" with the closed-end approach. A map prepared by teachers or domain experts are called "goal map," and a map built by a learner is called "learner map." Learners create their learner map with the parts composing a goal map. A set of components of the map is called "kit." This mechanism helps learners to build a concept map and make it possible to evaluate learner maps automatically in terms of the difference between learner maps and a goal map (Fig. 1).

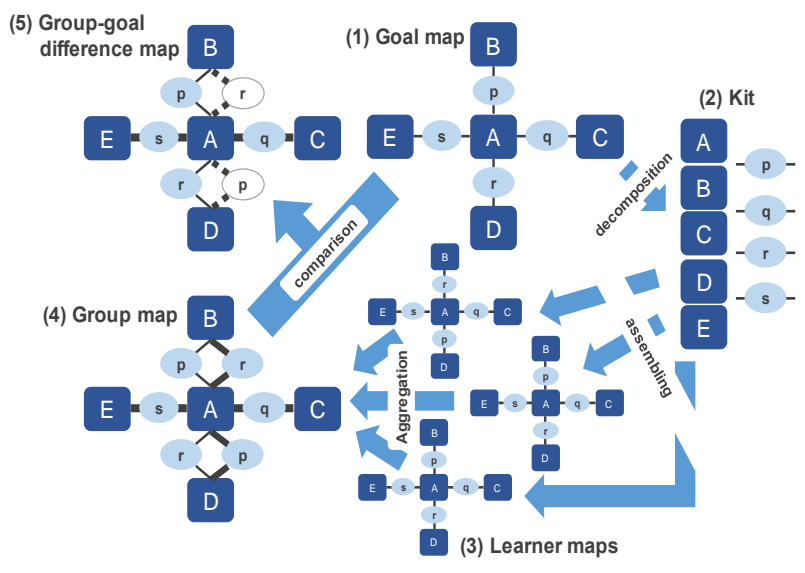

Fig. 1

Kit-build Concept Map and Diagnostic System

The KB map framework has two features. One is that the general concept map construction task consists of a segmentation task and a construction task. In the KB map framework, the recognition of components in a kit is the first task for concept map construction instead of the segmentation task. The goal map should be an ideal map that learners need to build. The applicable targets for KB maps are limited, and the learning environment requires some additional features. It is, therefore, necessary to propose an appropriate way to use KB maps under these restrictions.

KB maps can be used when using concept maps as a tool for confirming and reflecting learning or education already undertaken with clear learning/teaching goals. If you can specify a goal concept map, the map represents what the learner needs to acquire through learning or teaching. In such cases, the goal map portion must be 
explicitly displayed in the learning/teaching process. Therefore, recognizing them is a valuable activity in terms of both learning itself and the measurement of learning results. Since this is not a particular situation in learning/education, we believe that the scope of the $\mathrm{KB}$ map is sufficiently broad. Based on these considerations, the preparation of learning materials and goal maps is an essential phase in the KB map framework.

This paper introduces the mechanism and the effectiveness of the $\mathrm{KB}$ map framework and some case studies. The second chapter shows the validity of the automatic evaluation of the concept map in the KB map framework and the learning effect of the KB map. The third chapter explains the use cases of $\mathrm{KB}$ map in collaborative learning, English as a foreign language (EFL) learning. The last chapter concludes this paper.

\section{KIT-BUILD CONCEPT MAP}

\section{A. The Framework of Kit-Build Concept Map}

With KBmap, teachers create concept maps as a summary of learning concepts presented to learners. The validity of the concept map is guaranteed by the teacher. A goal map represents (1) the learner's expected understanding as a learning goal, and (2) the components that the teacher provides to show the learner an understanding. A kit is a decomposition of a goal map. The teacher gives the kit to the learner and asks them to create a concept map. Figures 1 shows the overview of KB map framework.

1. Learner Map

Learners assemble the components in the kit and create a concept map as a representation of understanding. Providing parts of concept maps helps learners first recognize the elements in the learning content and then understand the process of building them as an overall understanding of the learning content.

\section{Diagnosis and Feedback}

One of the features of KBmap is that the teacher can confirm the learner's understanding as to the difference between the goal map and the learner map. A learner map, composed of goal map components, allows comparisons between them. The difference represents the understanding gap between teachers and learners and learners.

The KBmap assessment method is automated and validated for assessing learner understanding [12]. In addition to one-to-one comparisons, learner maps can be overlapped as a representation of the learner's aggregated knowledge. Teachers can also compare overlapping maps with goal maps. Overlap maps allow teachers to analyze learner tendencies in class. This analysis helps teachers provide feedback to learners [13][14].

\section{KBmap System}

The kit build concept mapping system is called the "KBmap system." [15] The KBmap system consists of two client systems, "KBmap Editor" and "KBmap Analyzer," and the server system is "KBmap DB." The KBmap editor runs on a tablet computer, which allows the system to use in a usual classroom. Learners use the KBmap editor to create learner maps from the kit. Teachers evaluate learner maps using the KBmap Analyzer.

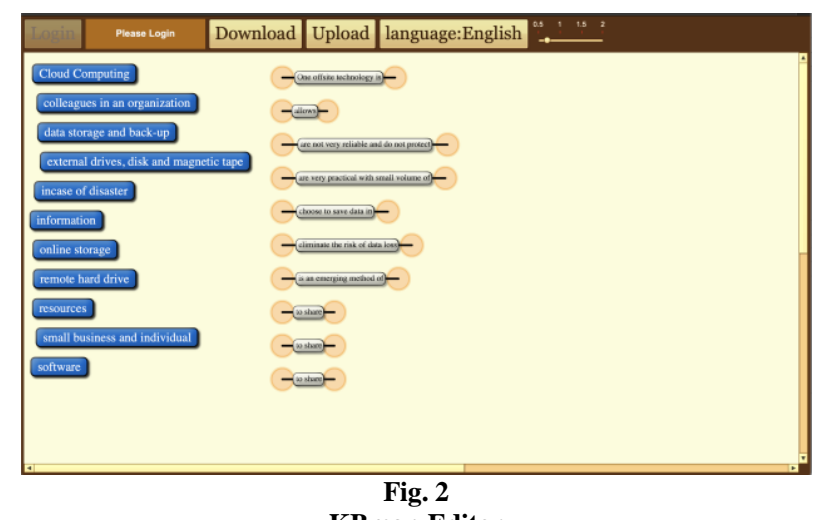

KBmap Editor

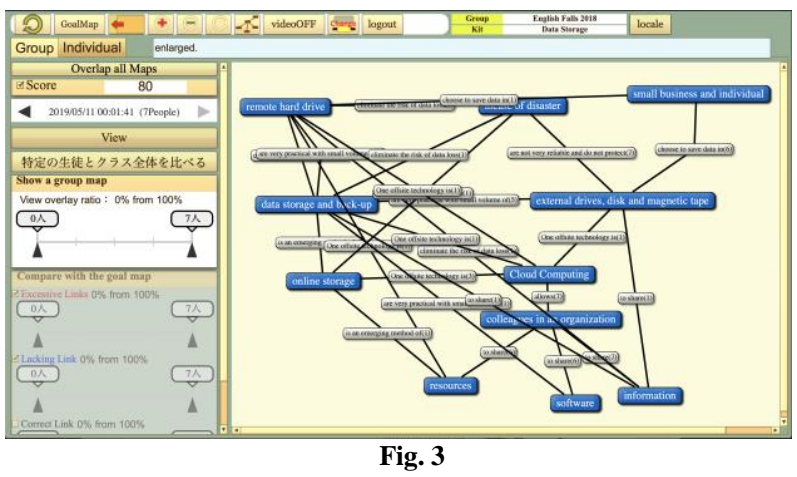

KBmap Analyzer

\section{B. The Validity and Effectiveness of KB Map}

1. The Validity of Automatic Assessment in KB Map

Wunnasri investigated the efficacy of automated evaluation of $\mathrm{KB}$ maps compared to manual evaluation methods [12]. She experimented with a case study to compare the results of this method with the results of two other evaluation methods. In this experiment, 22 university students participated as subjects and four as evaluators. The KB method was found to be very strongly correlated with other manual method scores. The results of this experiment are part of the evidence that the automated evaluation of KB maps can achieve almost the same level of effectiveness as well-known manual evaluation methods.

\section{The Learning Effects}

Funaoi et al. conducted an experiment comparing the KB map method with the general concept map method in terms of learner memory, focusing on "memory storage through concept map construction." [16] In the KB method, learners recognized the provided components of the concept map, and in the general method, learners identify components by themselves. The results showed 1) there is no memory difference between the KB method and the general method involving the content included in the kit. 2) the general method scores are higher for content not included in the kit. This result shows that segmentation is not always required, and the kit build method is useful for memory when well-structured teaching materials are prepared.

To investigate the value of the $\mathrm{KB}$ map task, Kitamura et al. compared it to the fill-in-the-blank (FIB) question with the multiple-choice [17]. Both tasks can be generated from the same concept map. For fifth-grade 
elementary school students, two classes were compared using three science lessons. One used KB maps, and the other used FIB questions. Learning effectiveness was assessed by writing tasks related to what students learned in the lessons. The results showed that the KB task evaluated learners' understanding better than the FIB question task, and the KB class performed better than the FIB class.

Hayashi et al. investigated the correlation between metacognitive ability and map scores [19]. Metacognitive ability consists of three sub-abilities, metacognitive monitoring, control, and knowledge. Correlation indicates which sub-abilities affect the map score or learner's understanding when learning with KB maps. The results show that there is a correlation between metacognitive control and map score. From this result, KB maps can be considered useful for learners to monitor cognition.

\section{LEARNING SUPPORT WITH KB MAP A. Formative Assessment}

Formative evaluation can encourage instructors to improve the learning outcomes of a lecture class. The goal of formative assessment in the classroom is to monitor learners and provide instructor feedback to enhance learner understanding and instructor expectations. The $\mathrm{KB}$ map is a digital tool that represents the instructor's expectations and supports a concept map strategy to assess learners' current understanding. The Kit-Build concept map is also suitable for implementing formative evaluation in lecture classes. A perfect propositional level match between the instructor and learner concept maps can generate diagnostic results and inform the instructor about the gap between current learner understanding and instructor expectations.

Instructors can, therefore, design feedback based on diagnostic results to improve learner understanding. In this paper, we propose an arrangement of KB map capabilities for formative evaluation in lecture classes to create opportunities to assess learners' current understanding whenever possible. Pairai et al. demonstrated the effectiveness of the kit build concept map for a closed-end approach in elementary school through three practical uses in various lecture classes [14].

The results of practical use showed that the functionality of the $\mathrm{KB}$ map could adjust the formative evaluation to fill the cycle as much as possible. The details of formative assessment may vary from instructor to instructor. The KB map has sufficient ability to contribute to the collection and evaluation of learner evidence and encourage the instructor to develop a positive situation in the classroom. The concept map strategy is used to create learning objectives for the class and to elicit learner understanding. You can use goal maps and learner maps to see the gap between the current understanding between instructors and learners about the same lecture content in the form of diagnostic results.

The learner's evidence diagnostic results (individual diagnostic results) and the learner's additional evidence (group diagnostic results) are practical information about the feedback design contributions of both in-class and inter-class feedback instructors. The KB map classroom environment, therefore, provides the opportunity to bridge the gap between current and desirable performance and provides information to instructors that can be used to shape lectures. These are the principles of good feedback practice [20].

Pairai et al. providing $\mathrm{KB}$ maps with tags with confidence [21]. The actual use was made to show valuable accuracy and reliability information in a lecture class. Accuracy information was visualized in the control group, and accuracy and reliability information was visualized in the experimental group. Observed evidence indicates that different information was used to select and order supplemental content when the system visualized different information. Normalized learning gains and effect sizes show different learning outcomes between the control and experimental classes. The results suggest that learner confidence information affects instructor behavior. The survey results suggest that KB maps with confidence tagging are an accepted mechanism for expressing learner understanding and confidence. The instructor also agreed that the learner's confidence information is valuable information for recognizing the learning situation.

\section{B. Adaptive Support}

To improve learner understanding in reading conditions, Pairai et al. proposed adaptive feedback for the KB map with confidence tagging [22]. Learners can construct a concept map to express their understanding as a learner map, and identify confidence in each proposition of the learning map as an understanding level. The KB map has already realized automatic diagnosis at the proposition level of the learner map.

Therefore, KB Map with confidence tagging uses both the accuracy and reliability information of each proposition to design and differentiate feedback, that is, (1) correct and confident, (2) correct and unconfident, (3) wrong and confidence, and (4) inaccurate and unconfident. An experiment was conducted to examine the effectiveness of adaptive feedback.

The results suggest that the learner can modify the map after properly receiving feedback. If you are "incorrect and unconfident," adaptive feedback can help improve your confidence. In the case of "inaccurate and confident," the improvement of the proposition was the same as in the case of "inaccurate and unconfident" Delayed test results show that learners can maintain understanding and confidence after a week.

\section{User Interface}

Building a computer-aided concept map from the provided pieces using a kit build is an activity that can facilitate understanding and retention. At the same time, users need to search for parts and organize the layout, which is thought to increase the overall cognitive load of the activity. Furtado et al. propose the Airmap interface that uses automatic layout management and spatial separation to improve the cognitive load when building a concept map [23].

Two experiments were conducted to create a concept map after participants $(\mathrm{N}=60, \mathrm{~N}=50)$ read the text. The results show that Airmap has succeeded in reducing cognitive load without a significant difference in immediate reading ability. However, there are significant differences in performance after a two week retention period. The results provide new insights into the retention 
enhancement aspect of closed concept map construction. The cognitive load reduced by the new interface is a close type and affects the depth at which memory users commit information.

\section{CASE STUDIES}

\section{A. Collaborative Learning}

\section{Knowledge Propagation}

Nomura et al. and Hayashi et al. propose a method to analyze the relationship between individual activities and group work in the classroom based on $\mathrm{KB}$ map [24][25]. Typically, classroom practice in a collaborative learning context revolves around three distinct levels of activity: individual work, group work, and class-wide discussion. It is essential to identify and analyze the correlations between these levels so that teachers can understand and improve student understanding dynamics in the context of collaborative learning.

Nomura et al. proposed a learning activity using $\mathrm{KB}$ maps in a group for building and sharing knowledge before the development of creativity, and investigated the knowledge transmission between students in the activity [24]. This result supports three hypotheses: "Students share their understanding through discussions," "Students" understanding approaches to the right one," and "Teachers can teach based on group maps." As a result, all possible patterns exist, and most of the propositions have been correct. From these results, students were able to acquire and correct knowledge through the proposed group work.

In the analysis of knowledge propagation, most students retain the correct knowledge and correct it through discussion. It indicates that the proposed method did not adversely affect students in this case. KB maps provide students with a common understanding of creating concept maps. This may be effective for discussion. When students create concept maps freely, it is challenging to organize their thoughts in a short time. This is a very controversial issue that needs further investigation.

Hayashi et al. classified groups in terms of knowledge propagation [25]. The result shows four types of groups in collaborative learning. Such data can be used for formative and summative evaluation. The teacher in this study was able to qualitatively assess the students' understanding, rather than quantitatively, and provide feedback accordingly. Typically, it is difficult for teachers to monitor the learning and comprehension process during collaborative learning. The conventional methods of acquiring this knowledge include allowing the students to give a presentation or carefully scrutinizing their conversation.

\section{Reciprocal Learning}

Collaborative learning is an active teaching and learning strategy, and learners who have detailed each other learn best. However, it is difficult for learners to explain their understanding to others in collaborative learning. Wunnasri et al. propose collaborative use of $\mathrm{KB}$ map called "Reciprocal KB map." [26] In the pair discussion with KB map, first, two participants create their concept maps that represent their level of understanding. Next, replace the map components and ask each other to use the parts to rebuild the map.

The difference between the original concept map and the rebuilt map is automatically diagnosed as an advantage of the $\mathrm{KB}$ map. A mutual $\mathrm{KB}$ map is expected to promote paired discussions, recognize mutual understanding, and create productive talks. In the experiments reported in this white paper, a mutual $\mathrm{KB}$ map was used to support pair discussions and compared with pair discussions supported by traditional concept maps.

Nineteen college students were required to use conventional concept maps in the discussion, and twenty college students used mutual KB maps to discuss the same topic. The results of the experiment were analyzed using three indicators: discussion score, similarity score, and questionnaire. A discussion score that examines the value of talk in discussions shows that a mutual $\mathrm{KB}$ map can facilitate more productive conversations between partners compared to traditional concept maps.

The similarity score, which evaluates the similarity of concept maps, shows that a discussion with KB map can help partner pairs better understand each other compared to traditional concept maps. Lastly, the survey indicates that the conversation with KB Map has supported this pairing of partners and has accepted this way of sharing their understanding. These results suggest that the Mutual KB Map is a promising approach for promoting mutual understanding and facilitating productive discussions among partner pairs (Fig. 4; Fig. 5).

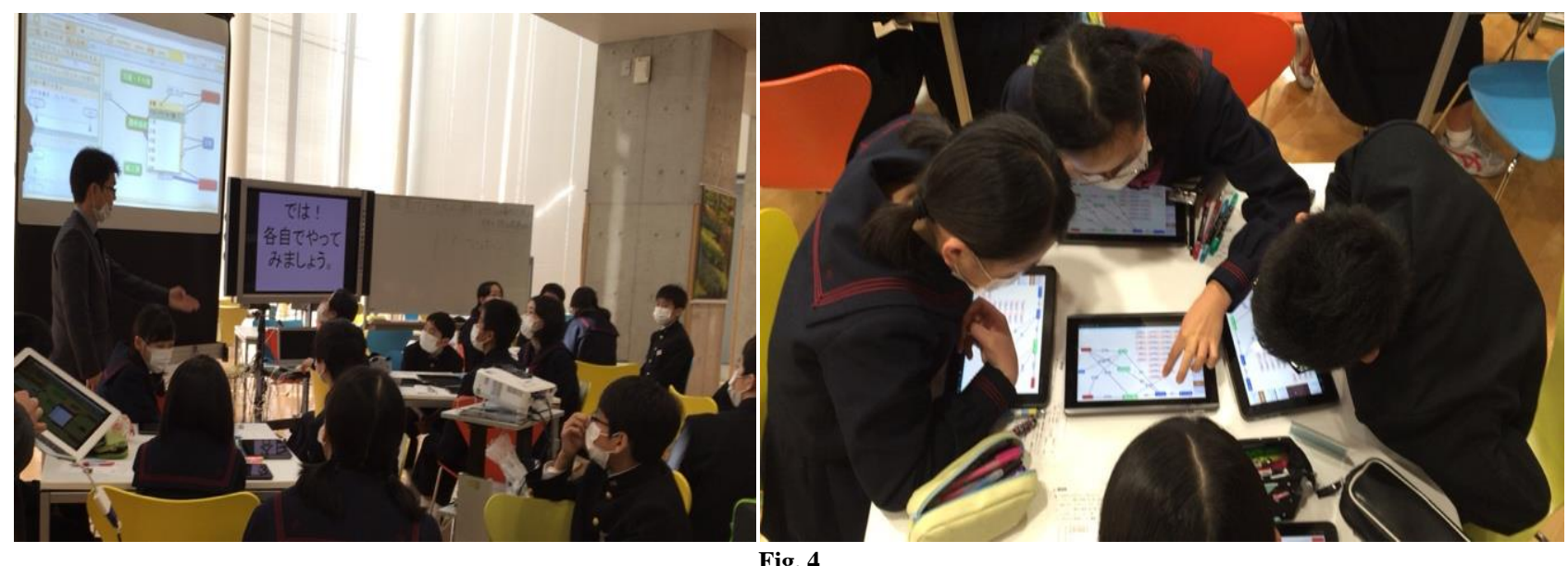

Pictures of Class: Group Work (Left) and Whole Class Discussion (Right) 


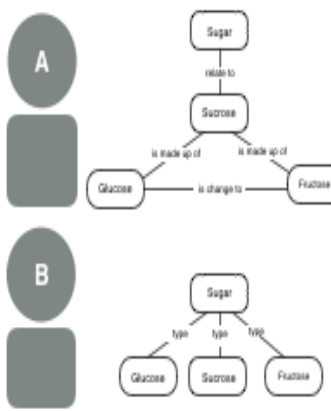

(1) $A$ and $B$ construct a concept map respectively
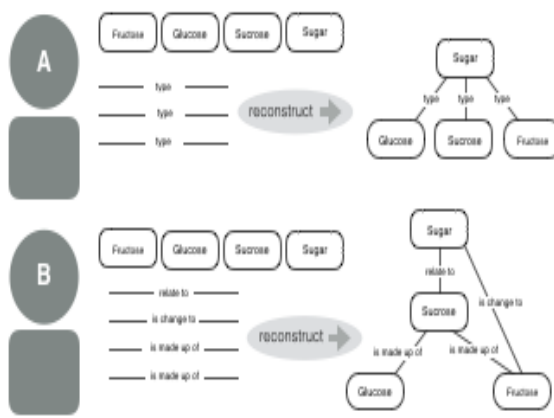

(2) A reconstructs a map with a kit of $B$ $B$ reconstructs a map with a kit of $A$

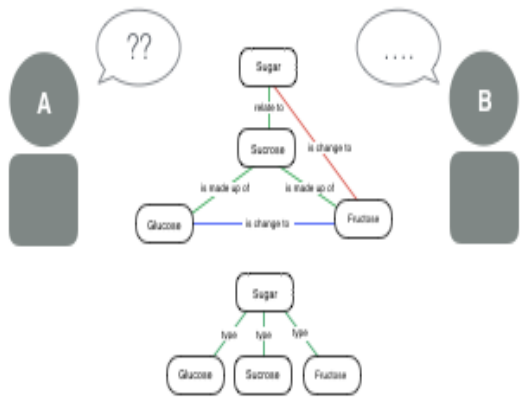

(3) A and B discuss about their same/different understandings by using two comparison maps

Fig. 5

Process of Reciprocal KB Map

\section{B. English as a Foreign Language Learning}

Reading comprehension is an essential but challenging task in language learning. English reading comprehension as a foreign language (EFL) context is a specific case of reading comprehension. This is a complex, dynamic, multicomponent, multidimensional task in the learning process. This is an ongoing process of multiple interactions between the reader's native language (ML) background knowledge and the knowledge published in the target language (TL), here English. Reading EFL is the same as reading ML. However, it is slower than ML reading and has a lower success rate [27].

$\mathrm{KB}$ map in EFL reading comprehension learning is more efficient than SB mapping in recalling understood information, as shown by the delayed comprehension test after two weeks [28]. KB mapping and SB mapping. The value out of the order of the sentence of KB mapping was higher than that of SB mapping. KB mapping can be thought of as facilitating learners with a mental model of textual content that is not based on sentence order.

Alkhateeb investigates the research question, "Why is the $\mathrm{KB}$ mapping method more efficient than the SB mapping method in recalling understanding information after two weeks?" [29]He experimented with comparing the progress of learner map construction using the $\mathrm{KB}$ mapping method and the SB mapping method.

The experiment monitored learner performance in terms of map size and process to made the concept map. Although SB mapping learners tended to use a sentenceby-sentence mapping style, KB mapping learners did not. Most low reading ability learners in EFL reading comprehension tend to do sentence-by-sentence reading [30]. The KB mapping technique helps learners to avoid the sentence-by-sentence map construction style and to escape from this style of mapping.

Andoko focuses on the paragraph structure of concept mapping [31]. One crucial element of reading comprehension is paragraphs [32], and there are many strategies for reading comprehension based on paragraphs. How much the map creation order differs from the order of the sentences in the text. The results show that KB mapping tends to deviate from the order of statements than SB mapping. However, it does not show how learners differ from the sentence order. In this study, from the perspective of paragraph structure, we will further analyze the concept mapping, specifically, whether the learner deviates randomly or in a specific pattern. The results show no significant difference in PR, but the former $A D$ is higher than the latter. Both types of reading pay attention to the paragraph as a unit of meaning, while reading with SB mapping tends to follow the text sentence for each sentence, but reading with KB mapping is independent of text Sentence order that tends to organize meaning.

Andoko et al. propose an approach to improve English reading comprehension with the help of KB-map with an additional function named source connection [33]. Fig. 6 shows the user interface of it. A university teacher in Indonesia practically used this approach and the other two approaches in several English classes of the second year of undergraduate students to compare the effectiveness of those approaches. The result shows that KB-map with the source-connection function was more effective in English reading comprehension compare to the traditional summarization and the standard Kit-build method (Fig. 6).

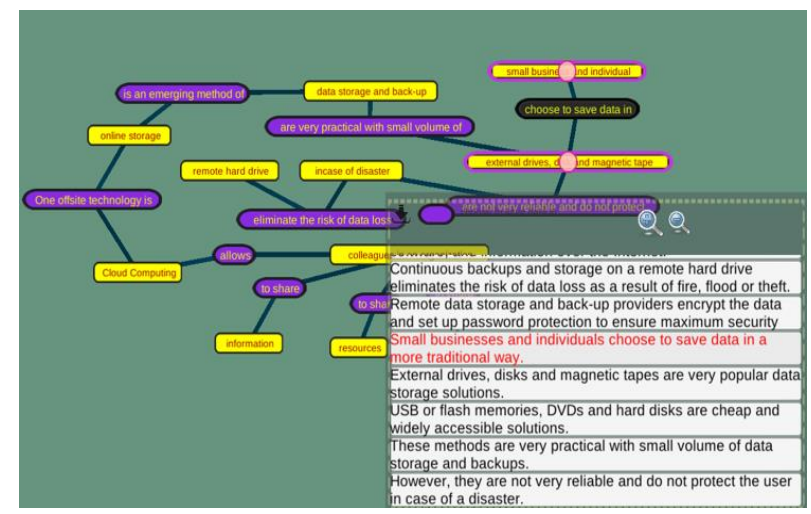

Fig. 6

KB Map with Source Connection

\section{CONCLUSION}

This paper shows an overview of the KB map framework and case studies. KB map is a concept map with the closed-end approach, and it enables the automated diagnosis of concept maps. These characteristics make it possible for teachers to conduct a formative assessment during a class and for learning environments to provide learners with adaptive feedback. By taking the advantages, we conducted many experiments and practical use of the KB map system to investigate the efficacy of it. 
A limitation of this study is that the target of the analysis in the KB map is only the result of concept map construction. Understanding of a topic in a learner has a potential impact on the construction process of a concept map of the topic. The analysis of the construction process of concept maps in the KB map has a potentially important implication for the analysis of understanding in learners.

\section{ACKNOWLEDGMENT}

I am grateful to Prof. Tsukasa Hirashima for the original idea of KB map and helpful discussions. I also gratefully acknowledge the work of past and present members of Learning engineering laboratory in the graduate school of engineering in Hiroshima university.

\section{REFERENCES}

[1] J.D. Novak, and D.B. Gowin, Learning how to learn. New York, NY: Cambridge University Press, 1984.

[2] H. Barenholz, and P. Tamir, "A comprehensive use of concept mapping in design, instruction and assessment," Science and Technological Education, 10, 1992, pp. 37-52.

[3] A. Ozdemir, "Analyzing concept maps as an assessment (evaluation) tool in teaching mathematics," J Soc Sci, vol. 1, no. 3, 2005, pp. 141-149.

[4] K. Chang, T. Sung, and S.F. Chen, "Learning through computer-based concept mapping with scaffolding aid, "J Comput Assist Learn, vol. 17, no. 1, 2001, pp. 21-33.

[5] L. Cimolino, and J. Kay, "Verified concept mapping for eliciting conceptual understanding," Proceedings of ICCE2002, 2002, pp. 1561-1563.

[6] T. Conlon, T, “'But is our concept map any good?' Classroom experiences with the reasonable fallible analyser," Proc. of the First Int. Conference on Concept Mapping.

[Online]. Available: http://cmc.ihmc.us/papers/cmc2004-054.pdf.

[7] E. Gouli, A. Gogoulou, K.A. Papanikolaou and M. Grigoriadou, "Evaluating learner's knowledge level on concept mapping tasks," Proceedings of Fifth IEEE International Conference on Advanced Learning Technologies, 2005, pp. 424-428.

[8] E. Taricani and R. Clariana, "A technique for automatically scoring open-ended concept maps," Educ Technol Res Dev, vol. 54, no. 1, 2006, pp. 65-82.

[9] K. Chang, T. Sung and S.F. Chen, "Learning through computer-based concept mapping with scaffolding aid,"J Comput Assist Learn, vol. 17, no. 1, 2001, pp.21-33.

[10] J.D. Novak and A.J. Cañas, "The theory underlying concept maps and how to construct them," Technical Report IHMC CmapTools 2006-01 Rev 01-2008. 2008.

[11] B.S. Bloom, M.D. Engelhart, E.J. Furst, W.H. Hill and D.R. Krathwohl, "Taxonomy of educational objectives: the classification of educational goals," Handbook I: cognitive domain. New York: David McKay Company, 1956.

[12] W. Wunnasri, J. Pailai, Y. Hayashi and T. Hirashima, "Validity of Kit-Build Method for Assessment of LearnerBuild Map by Comparing with Manual Methods", IEICE Transactions on Information and Systems, Vol.E101-D(4), 2018, pp. 1141-1150.

[13] K. Yoshida, K. Sugihara, Y. Nino, M. Shida and T. Hirashima, "Practical use of Kit-Build concept map system for formative assessment of learners' comprehension in a lecture," Proc. ICCE 2013, 2013, pp. 906-915.

[14] J. Pailai, W. Wunnasri, K. Yoshida, Y. Hayashi and T. Hirashima, "The practical use of Kit-Build concept map on formative assessment," Research and Practice in
Technology Enhanced Learning vol. 12:20, 2017. https://doi.org/10.1186/s41039-017-0060-x.

[15] K. Sugihara, T. Osada, S. Nakata, H. Funaoi, and T. Hirashima, "Experimental evaluation of Kit-Build concept map for science classes in an elementary school," Proc. ICCE 2012, 2012, pp. 17-24.

[16] H. Funaoi, K. Ishida, and T. Hirashima, "Comparison of Kit-Build and Scratch-Build Concept Mapping Methods on Memory Retention,” Proc. of ICCE 2011, 2011, pp. 539546.

[17] T. Kitamura, A. Yamanaka, K. Maeda, Y. Hayashi, and T. Hirashima, "Learning Task Generation from a Series of Propositions of a Learning Topic: Kit-Building Task of Concept Map and Multiple Choice Task of Fill-in-theblank Questions," Proc. 24th Int. Conf. on Computers in Education, India, pp.91-93, 2016.

[18] T. Kitamura, Y. Hayashi, T. Hirashima, "Generation of Fill-in-the-Blank Questions from Concept Map and Preliminary Comparison between Multiple-Choice Task and Kit-Build Task," The Journal of Information and Systems in Education, vol. 18, no. 1, 2019, pp. 11-15.

[19] Y. Hayashi and T. Hirashima, "Analysis of the Relationship Between Metacognitive Ability and Learning Activity with Kit-Build Con- cept Map,” Proc. Int. Conf. on Human Interface and the Manage- ment of Information, United States, 2015, pp.304-312.

[20] D.J. Nicol and D. Macfarlane-Dick, "Formative assessment and self-regulated learning: A model and seven principles of good feedback practice," Studies in Higher Education, vol. 31, no. 2, 2006, pp. 199-218.

[21] J. Pailai, W. Wunnasri, Y. Hayashi and T. Hirashima, "KitBuild Concept Map with Confidence Tagging in Practical Uses for Assessing the Understanding of Learners," International Journal of Advanced Computer Science and Applications, vol. 9, no. 1, 2018.

[22] J. Pailai, W. Wunnasri, Y. Hayashi And T. Hirashima, "Correctness- and Confidence-based Adaptive Feedback of Kit-Build Concept Map with Confidence Tagging," Proc. of AIED2018, 2018, pp. 395-408.

[23] P.G.F. Furtado, T. Hirashima, Y. Hayashi, "Reducing Cognitive Load during Closed Concept Map Construction and Consequences on Reading Comprehension and Retention," IEEE Transactions on Learning Technologies, 2018.

[24] T. Nomura, Y. Hayashi, Y., T. Suzuki, T. and T. Hirashima, "Knowledge propagation in practical use of KitBuild concept map system in classroom group work for knowledge sharing," Proc. Int. Conf. on Computers in Education Workshop 2014, 2014, pp.463-472.

[25] Y. Hayashi, T. Nomura and T. Hirashima: Orchestrating Individual- and group-learning in Classroom with Kit-Build Concept Mapping," The 20th International Conference on Artificial Intelligence in Education (AIED2019), 2019, pp.100-104.

[26] W. Wunnasri, J. Pailai, Y. Hayashi, T. Hirashima, "Reciprocal Kit-Build Concept Map: An Approach for Encouraging Pair Discussion to Share Each Other's Understanding," IEICE Transactions on Information and Systems, vol. E101-D, no. 9, 2018, pp.2356-2367.

[27] N. Anderson, "Exploring Second Language Reading Issues and Strategies," Heinle \& Heinle Publishers, Boston, MA, 1999.

[28] M. Alkhateeb, Y. Hayashi, T. Rajab, and T. Hirashima, "Comparison between Kit-Build and Scratch-Build concept mapping methods in supporting EFL reading comprehension," The Journal of Information and Systems in Education, vol.14, no.1, 2015, pp.13-27.

[29] M. Alkhateeb, Y. Hayashi, T. Rajab, and T. Hirashima, "Experimental Evaluation of the KB-mapping Method to Avoid Sentence-by-Sentence Map-building Style in EFL 
Reading with Concept Mapping," Information And Systems In Education, vol. 15, no. 1, 2016, pp.1-14.

[30] E. Block, "The Comprehension Strategies of Second Language Readers," TESOL Quarterly, vol. 20, no. 3, 1986, pp. 463-494.

[31] B.S. Andoko, Y. Hayashi, T. Hirashima, "Analysis of the concept mapping style in EFL reading comprehension comparison between kit-build and scratch-build concept mapping from the viewpoint of paragraph structure of text," Science in Information Technology (ICSITech), 2017, pp. 622-625.

[32] E. Thorndike, "Reading as Reasoning: A Study of Mistakes in Paragraph Reading," Reading Research Quarterly, vol. 6, no.4, 1971, pp. 425-434

[33] B.S. Andoko, Y. Hayashi, T. Hirashima, "Reading Assistance for EFL Readers with Kit-build Concept Map with Source-connection," The 27th International Conference on Computers in Education (ICCE 2019), 2019 ,

to

appear. 